International Journal of Uncertainty,

Fuzziness and Knowledge-Based Systems

Vol. 24, No. 6 (2016) 965-968

(C) World Scientific Publishing Company

DOI: $10.1142 / \mathrm{S} 0218488516990014$

\title{
Author Index \\ Volume 24 (2016)
}

Adhami-Mirhosseini, A., see Sheikhlar, A.

24 (2016) 761

Aggarwal, S. \& Gupta, C., Solving intuitionistic fuzzy solid transportation problem via new ranking method based on signed distance

Araabi, B. N., see Sarabi-Jamab, A.

24 (2016) 483

24 (2016) 503

Barman, B., Kanjilal, R. \& Mukhopadhyay, A., Neuro-fuzzy controller design to navigate unmanned vehicle with construction of traffic rules to avoid obstacles

24 (2016) 433

Beik-Mohammadi, H., see Sheikhlar, A.

Cabañas, R., Gómez-Olmedo, M. \& Cano, A., Using binary trees for the evaluation of influence diagrams

24 (2016) 761

$24(2016) 59$

Cano, A., see Cabañas, R.

Chen, H., see Liu, X.

24 (2016) 59

24 (2016) 593

Chen, R., see Lin, J.

24 (2016) 873

Chen, X., Wang, Y., Zhen, S., Huang, K., Zhao, H. \& Chen, Y.-H., Robust control design of uncertain mechanical systems: a fuzzy approach

24 (2016) 307

Chen, Y.-H., see Chen, X.

24 (2016) 307

Chen, Z., see Zong, G.

24 (2016) 387

Deng, Y., see He, Y.

Deng, Y., see Mo, H.

24 (2016) 13

24 (2016) 831

Dong, J.-Y., Lin, L.-L., Wang, F. \& Wan, S.-P., Generalized Choquet integral operator of triangular Atanassovs intuitionistic fuzzy numbers and application to multi-attribute group decision making

$24(2016) 647$

Dorri Nogoorani, S. \& Jalili, R., Uncertainty in trust: a risk-aware approach

Fakharian, A., see Sheikhlar, A.

24 (2016) 703

24 (2016) 761

24 (2016) 367

Ghansah, B. \& Wu, S., A mean-variance analysis based approach for search result diversification in federated search

24 (2016) 195

Gupta, C., see Aggarwal, S.

24 (2016) 483

Guzmán, V. C., Masegosa, A. D., Pelta, D. A. \& Verdegay, J. L., Fuzzy models and resolution methods for covering location problems: an annotated bibliography

24 (2016) 561

Gómez-Olmedo, M., see Cabañas, R.

24 (2016) 59 
He, Y., He, Z., Zhou, P. \& Deng, Y., Scaled prioritized geometric aggregation operators and their applications to decision making

He, Z., see He, Y.

Hokari, T. \& Yao, M., Eliciting subjective probabilities in Anscombe and Aumann's model

Hong, D. H., Berwald and favard type inequalities for fuzzy integrals

Hu, C.-F., see Hu, C.-K.

Hu, C.-K., Liu, F.-B. \& Hu, C.-F., A set covering-based diagnostic expert system to economic and financial applications

Huang, H.-C. \& Yang, X., A comparative investigation of type-2 fuzzy sets, nonstationary fuzzy sets and cloud models

Huang, K., see Chen, X.

Huo, W., Feng, X. \& Zhang, Z., An efficient approach for incremental mining fuzzy frequent itemsets with FP-tree

Jalili, R., see Dorri Nogoorani, S.

Jana, D. K., Pramanik, S. \& Maiti, M., A parametric programming method on Gaussian type-2 fuzzy set and its application to a multilevel supply chain

Jiang, J.-N., see Yao, L.

Jiang, Z., see Rao, D.

Kanjilal, R., see Barman, B.

Lan, Y., see Zong, G.

Lei, Q., see Tian, F.

Li, X.-Z., see Liu, Y.

Lin, J., Chen, R. \& Zhang, Q., Similarity-based approach for group decision making with multi-granularity linguistic information

Lin, L.-L., see Dong, J.-Y.

Lin, T.-B., see Yao, L.

Liu, F.-B., see $\mathrm{Hu}$, C.-K.

Liu, J., see Zhou, J.

Liu, S., see Tian, F.

Liu, X., Tao, Z., Chen, H. \& Zhou, L., A MAGDM method based on 2-tuple linguistic heronian mean and new operational laws

Liu, Y., Li, X.-Z. \& Zhang, Y.-Y., Random fuzzy repairable coherent systems with independent components

Maiti, M., see Jana, D. K.

Martin, A., see Zhou, K.

Martinez-Gil, J., Accurate semantic similarity measurement of biomedical nomenclature by means of fuzzy logic

Masegosa, A. D., see Guzmán, V. C.

Mehta, R., see Radhakrishnan, B. M.

Min, Y.-M., see Qin, F.

Miranda, E., see Pelessoni, R.

Mishra, S. \& Srivastava, R., Representability of fuzzy biorders and fuzzy weak order

24 (2016) 13

24 (2016) 13

24 (2016) 405

24 (2016) 47

24 (2016) 91

$24(2016) 91$

24 (2016) 213

24 (2016) 307

24 (2016) 367

24 (2016) 703

24 (2016) 451

24 (2016) 137

24 (2016) 167

24 (2016) 433

24 (2016) 387

24 (2016) 631

24 (2016) 859

24 (2016) 873

24 (2016) 647

24 (2016) 137

24 (2016) 91

24 (2016) 537

24 (2016) 631

24 (2016) 593

24 (2016) 859

24 (2016) 451

24 (2016) 937

24 (2016) 291

24 (2016) 561

24 (2016) 781

24 (2016) 685

24 (2016) 229

Mo, H. \& Deng, Y., A new aggregating operator for linguistic information based on D numbers

24 (2016) 917

$24(2016) 831$ 
Montes, I., see Pelessoni, R.

24 (2016) 229

Mukhopadhyay, A., see Barman, B.

24 (2016) 433

Narukawa, Y., see Torra, V.

Nettleton, D. F. \& Salas, J., Approximate matching of neighborhood subgraphs: an ordered string graph levenshtein method

24 (2016) 847

24 (2016) 411

Niknafs, A., see Saeed, S.

Pan, Q., see Zhou, K.

Pelessoni, R., Vicig, P., Montes, I. \& Miranda, E., Bivariate $p$-boxes

Pelta, D. A., see Guzmán, V. C.

Pramanik, S., see Jana, D. K.

24 (2016) 123

24 (2016) 937

24 (2016) 229

24 (2016) 561

24 (2016) 451

Qin, F. \& Min, Y.-M., Distributivity for semi-nullnorms over semi-t-operators

Qin, X., see Zhang, C.

Radhakrishnan, B. M., Srinivasan, D. \& Mehta, R., Fuzzy-based multi-agent system for distributed energy management in smart grids

Rao, D. \& Jiang, Z., Cost-sensitive action model learning

Saeed, S. \& Niknafs, A., Artificial bee colony-fuzzy Q learning for reinforcement fuzzy control (truck backer-upper control problem)

Salas, J., see Nettleton, D. F.

Sarabi-Jamab, A. \& Araabi, B. N., Information-based evaluation of approximation methods in Dempster-Shafer theory

Shao, Q., see Zhai, J.

Sharma, B., see Verma, R.

24 (2016) 685

24 (2016) 739

24 (2016) 781

24 (2016) 167

24 (2016) 123

24 (2016) 411

24 (2016) 503

24 (2016) 327

24 (2016) 265

Sheikhlar, A., Fakharian, A., Beik-Mohammadi, H. \& Adhami-Mirhosseini, A., Design and implementation of self-adaptive PD controller based on fuzzy logic algorithm for omni-directional fast robots in presence of model uncertainties

Srinivasan, D., see Radhakrishnan, B. M.

Srivastava, R., see Mishra, S.

Tao, Z., see Liu, X.

Tian, F., Liu, S., Xu, Z. \& Lei, Q., Diagram illustrations of aggregation operations for the intuitionistic fuzzy values

Torra, V., Narukawa, Y. \& Yager, R. R., On a relationship between fuzzy measures and AIFS

Türkşen, Ö., Analysis of response surface model parameters with Bayesian approach and fuzzy approach

Verdegay, J. L., see Guzmán, V. C.

Verma, R. \& Sharma, B., Prioritized information fusion method for triangular fuzzy information and its application to multiple attribute decision making

24 (2016) 761

24 (2016) 781

24 (2016) 917

24 (2016) 593

24 (2016) 631

$24(2016) 847$

24 (2016) 109

24 (2016) 561

Vicig, P., see Pelessoni, R.

24 (2016) 265

24 (2016) 229

24 (2016) 647

Wan, S.-P., see Dong, J.-Y.

24 (2016) 647

Wang, F., see Dong, J.-Y.

24 (2016) 901

Wang, H., Fubini theorems for capacities

24 (2016) 537

Wang, K., see Zhou, J.

24 (2016) 327

Wang, X., see Zhai, J. 
Wang, Y., see Chen, X.

24 (2016) 307

Wu, S., see Ghansah, B.

24 (2016) 195

Xin, Y.-K., see Zhang, Q.-H.

24 (2016) 347

$\mathrm{Xu}, \mathrm{Z}$., see Tian, F.

24 (2016) 631

$\mathrm{Xu}, \mathrm{Z}$., Additive intuitionistic fuzzy aggregation operators based on fuzzy measure

$24(2016) 1$

$\mathrm{Xu}, \mathrm{Z}$.-S., see $\mathrm{Yu}, \mathrm{S}$.

Yager, R. R., see Torra, V.

Yang, X., see Huang, H.-C.

Yao, L., Jiang, J.-N. \& Lin, T.-B., Observer based adaptive fuzzy controller with modulated membership functions for nonlinear system

24 (2016) 807

24 (2016) 847

24 (2016) 213

Yao, L.-Y., see Zhang, Q.-H.

Yao, M., see Hokari, T.

Yi, X., see Zhou, J.

Yu, S. \& Xu, Z.-S., An approach based on definite integrals to multi-criteria decision making with correlative intuitionistic fuzzy information

24 (2016) 807

Zadeh, L. A., A very simple formula for aggregation and multicriteria optimization

24 (2016) 961

Zhai, J., Shao, Q. \& Wang, X., Improvements for P-ELM1 and P-ELM2 pruning algorithms in extreme learning machines

Zhang, C., Zhang, J., Qin, X. \& Zhang, S., Miner*: a weighted distance sum based outlier mining system of star spectrum data

Zhang, G.-S., see Zhang, Q.-H.

Zhang, J., see Zhang, C.

Zhang, Q., see Lin, J.

Zhang, Q.-H., Yao, L.-Y., Zhang, G.-S. \& Xin, Y.-K., The incremental knowledge acquisition based on Hash algorithm

24 (2016) 137

24 (2016) 347

24 (2016) 405

24 (2016) 537

Zhang, S., see Zhang, C.

Zhang, Y.-Y., see Liu, Y.

Zhang, Z., see Huo, W.

Zhao, H., see Chen, X.

Zhen, S., see Chen, X.

Zhou, J., Yi, X., Wang, K. \& Liu, J., Uncertain distribution-minimum spanning tree problem

Zhou, K., Martin, A. \& Pan, Q., The belief noisy-OR model applied to network reliability analysis

Zhou, L., see Liu, X.

Zhou, P., see He, Y.

Zong, G., Chen, Z. \& Lan, Y., Fubini-like theorem of real-valued

Choquet integrals for set-valued mappings

$24(2016) 327$

24 (2016) 739

24 (2016) 347

24 (2016) 739

24 (2016) 873

24 (2016) 347

24 (2016) 739

24 (2016) 859

24 (2016) 367

24 (2016) 307

24 (2016) 307

$24(2016) 537$

24 (2016) 937

24 (2016) 593

24 (2016) 13

24 (2016) 387 